\title{
Surface rupture in stochastic slip models
}

\author{
S. Murphy ${ }^{1}$ and A. Herrero ${ }^{2}$ \\ ${ }^{1}$ IFREMER, Geoscience Marine, Plouzané, France.E-mail: shane.murphy@ifremer.fr \\ ${ }^{2}$ Istituto Nazionale di Geofisica e Vulcanologia, 00143 Rome, Italia
}

\begin{abstract}
SUMMAR Y
As an alternative to spectral methods, stochastic self-similar slip can be produced through a composite source model by placing a power-law scaling size-frequency distribution of circular slip dislocations on a fault surface. However these models do not accurately account for observed surface rupture behaviour. We propose a modification to the composite source model that corrects this issue. The advantage of this technique is that it accommodates the use of fractal slip distributions on non-planar fault surfaces. However to mimic a surface rupture using this technique, releasing the boundary condition at the top of the fault, we observed a systematic decrease in slip at shallow depths. We propose a new strategy whereby the surface is treated like a reflector with the slip being folded back onto the fault. Two different techniques based on this principal are presented: the first is the method of images. It requires a small change to pre-existing codes and works for planar faults. The second involves the use of a multistage trilateration technique. It is applied to non-planar faults described by an unstructured mesh. The reflected slip calculated using the two techniques is near identical on a planar fault, suggesting they are equivalent. Applying this correction, where reflected slip is accounted for in the composite source model, the lack of slip at shallow depths is not observed any more and there is no systematic trend with depth. However, there are other parameters which may affect the spatial distribution of slip across the fault plane. For example, the type of probability density function used in the placement of the subevent is also important. In the case where the location of maximum slip is known to a first order, a Gaussian may be appropriate to describe the probability function. For hazard assessment studies a uniform probability density function is more suitable as it provides no underlying systematic spatial trend.
\end{abstract}

Key words: Numerical approximations and analysis; Self-organization; Theoretical seismology.

\section{INTRODUCTION}

The production of rapid, realistic heterogeneous slip distributions is essential in many applications. The linking of the stress drop with power law scaling (Hanks 1979) and self-similar/self-affine slip distributions with seismic radiation (Andrews 1980) provided a means to producing stochastic slip distributions based on generic features of earthquakes. Herrero \& Bernard (1994) demonstrated that such distributions could be produced efficiently in the wavenumber domain using a limited number of parameters and a fast Fourier transform. Since then, a number of different techniques for producing self-similar/self-affine slip distributions with nuanced differences based on particular observations have been produced (e.g., Mai \& Beroza 2002; Lavallée \& Archuleta 2003; Song \& Somerville 2010; Goda et al. 2015; Davies et al. 2015). However, the effect the surface boundary has on slip are rarely discussed (e.g. Shimazaki 1986), particularly in the case of stochastic source models. Wells \& Coppersmith (1994) found that slip at depth and at the surface were similar for most earthquakes in their database. It is this feature that we aim to reproduce in our stochastic models.

Generally, in order to avoid singularities at the fault boundary, the slip is constrained through a selective choice of phases in the frequency space or is tapered to zero (e.g. Herrero \& Bernard 1994). In the case where a rupture reaches and breaks the surface, a simple solution, to take into account this phenomena, is to extend the fault plane above the surface in the air. The slip distribution is generated on this virtual fault, and afterwards the section above the surface is removed and the slip rescaled. Alternatively a careful application of the phases can be used to position the maximum slip near the surface. None of these solutions are easy to apply to non-planar fault surfaces.

In order to address this issue we use a composite source model (Frankel 1991; Zeng et al. 1994) to generate stochastic slip distributions. The advantage of this technique is that it is performed solely in the spatial domain requiring no fast Fourier transforms and therefore the fault surface may be described by an unstructured 


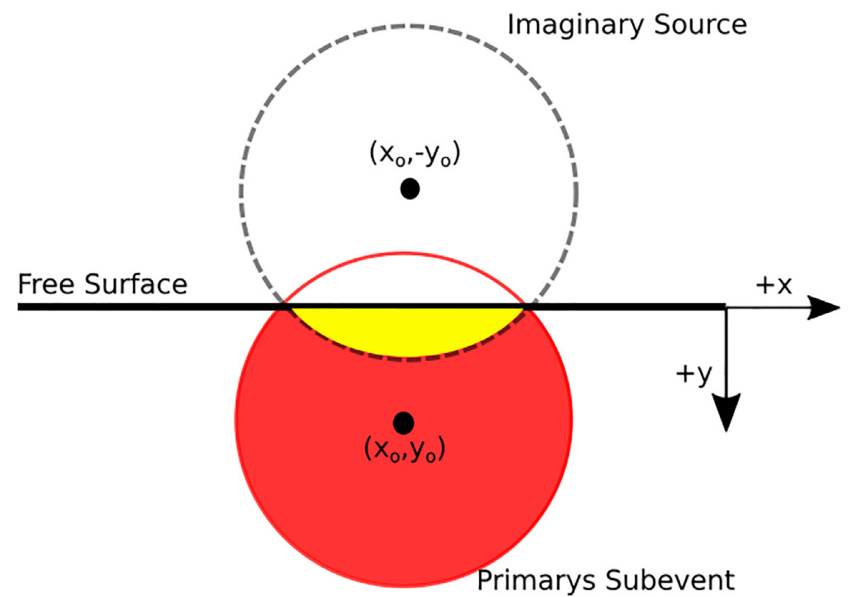

Figure 1. Reflecting slip back onto the fault plane using the method of images. The radial distance from the imaginary source is given by eq. (2). The red circle is the original subevent and the dash black line is the imaginary source. The zone in yellow marks the slip contribution from the imaginary source to the original subevent. (a)

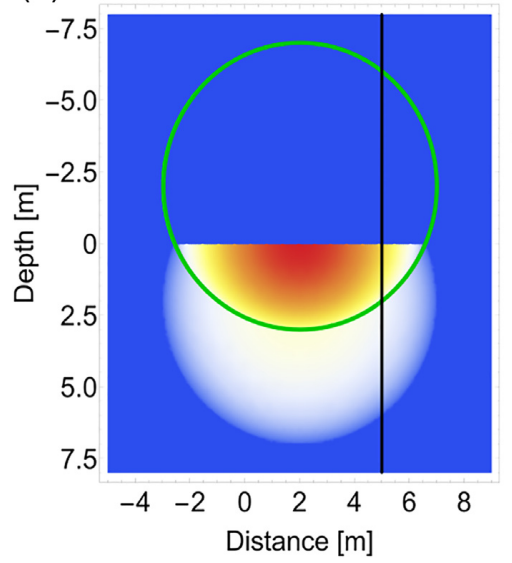

(b)

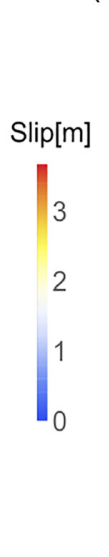

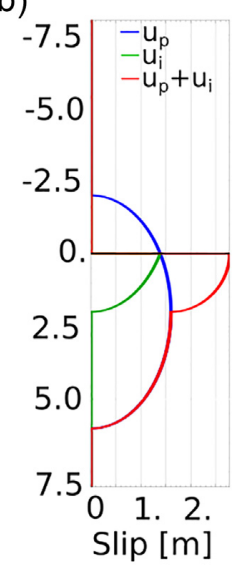

Figure 2. (a) 2-D subevent with a radially dependent slip distribution on a planar fault containing the reflected contribution. The slip profile of the primary subevent is centred at $(2,2)$ with the free surface located at $y=0$. The black line represents location of the cross-section shown in subplot $b$. (b) A cross-section of slip shown in subplot (a) decomposed into the primary subevent $u_{p}(r)$ in green, the imaginary contribution $u_{i}\left(r^{\prime}\right)$ in blue and the total slip $\left[u_{p}(r)+u_{i}\left(r^{\prime}\right)\right]$ in red.

mesh. This method involves the placement of a large number of circular subevents, with self-similar distribution in size, onto the fault surface. Their distribution is based on a pre-defined spatial probability density function. The summation of all the subevents produces a final slip distribution which exhibits a self-similar spectra. This is equivalent to $k^{-2}$ slip distributions (Ruiz et al. 2007). Herrero \& Murphy (2018) extended the use of the composite source model from planar to non-planar surfaces by using a double trilateration technique to calculate the distance across unstructured grids. An advantage of these methods is their capacity to guarantee null slip at the boundary of the fault. This is achieved by redeploying subevents whose radii extend outside the edge of the fault plane. If such a manoeuvre is applied along the top of the fault this will result in no surface rupture.

The inclusion of large surface rupture is challenging. Loosening the boundary condition along the free surface, so that subevent (a)

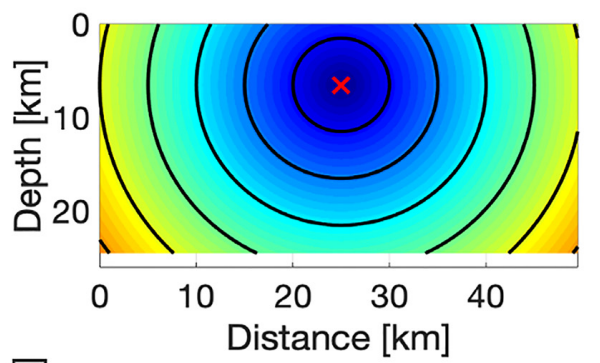

(b)

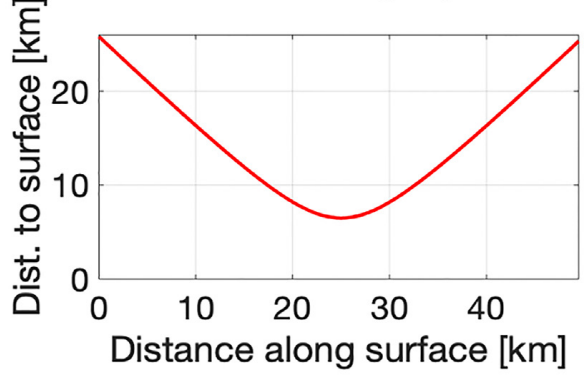

(c)

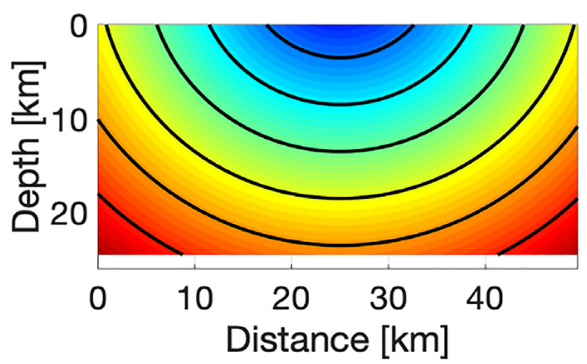

Figure 3. The steps in calculating the reflection from the surface. Panel (a) is the distance from the point source, denoted by the red $x$ to all points on the fault plane. The black contours are at $5 \mathrm{~km}$ intervals. The free surface is represented by nodes at $y=0$. Panel (b) is the distance from the point source in subplot a to all the surface nodes. (c) Setting the distance to zero at all nodes across the mesh with the exception of the surface nodes which retain the values calculated in the original step and plotted in subplot (b), the reflected distance is now calculated across the mesh.

which extend above the surface are not rejected, does produce slip at the surface. However it is systematically lower compared with slip at depth, a feature that will be demonstrated and explained in more detail in the following sections. Another approach is to extend the fault plane above the surface of the earth. Then, the slip is placed on the extended fault and the protruding part of the fault is removed afterwards (Murphy et al. 2016). This generates a smoother depth dependent function. However it requires a number of additional computational steps and, in the case of non-planar faults, it may be very difficult to apply.

Surface ruptures with systematic small values are at odds with a number of numerical and seismological observations. Originally earthquakes with surface slip were modelled as mirrored cracklike dislocations (Knopoff 1958) where the initial fault width was double and centred on the free surface, the fault plane was then halved and the slip doubled. This results in maximum slip always being located at the surface with the implicit assumption that the healing phase is generated from edges of the fault and not at the free surface. Heaton (1990) has since proposed that rupture may be pulse-like rather than crack-like in nature implying that a healing phase may be created before the rupture front reaches the fault edge. This has a collateral effect: the largest slip in an earthquake may occur anywhere on the fault. Dynamic simulations have shown that dipping thrust faults produce larger amounts of slip when compared 

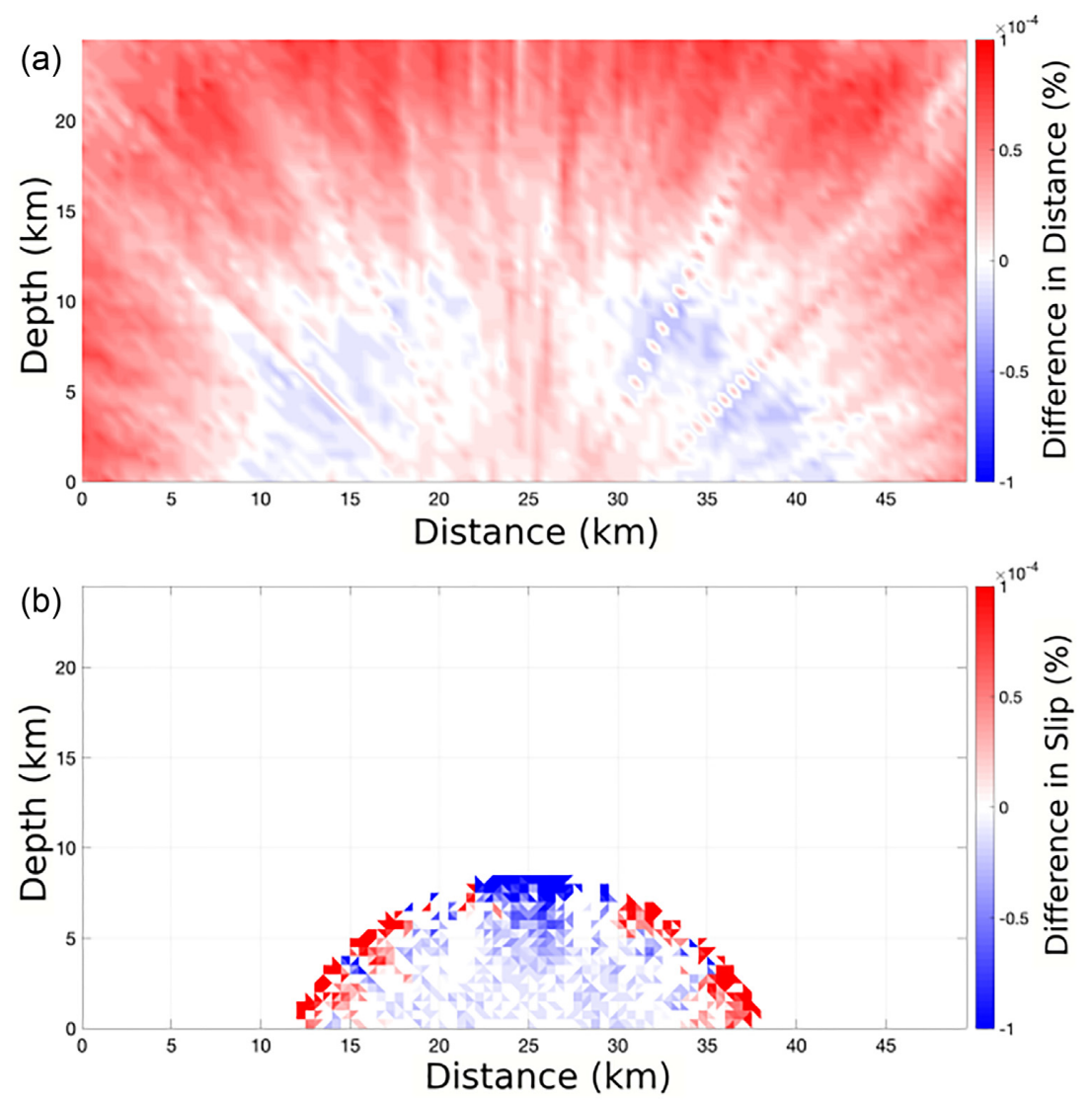

Figure 4. The difference in reflected distance/secondary slip when using the method of images compared to the MSTT. A subevent has been placed at a distance of $25 \mathrm{~km}$ along strike and $6.5 \mathrm{~km}$ depth with a radius of $15 \mathrm{~km}$. (a) The colour bar depicts the percentage difference in the reflected distance between the two techniques divided by the method of images distance [i.e. $100 \times\left(r_{M O I}-r_{M S T T}\right) / r_{M O I}$ where $r_{M O I}$ and $r_{M S T T}$ is the distance calculated using the method of images and MSTT techniques, respectively]. The calculated values are at the level of numerical noise. In subplot (b) the difference in the reflected slip distribution (i.e. secondary source) using the same subevent location as in subplot (b) is divided by the method of images slip (i.e. similar to the equation used for the distance in subplot (a). The difference between the two techniques is close to numerical noise.

with normal faulting due to seismic waves being reflected onto the fault during dynamic rupture (Nielsen 1998; Oglesby et al. 1998). Murphy et al. (2016) observed that the Japan Trench is susceptible to slip amplification at shallow depths while Scala et al. (2019a) demonstrated that seismic radiation interaction with rupture causes increased slip-rate and slip near the surface. Additionally slip inversions for a number of tsunamiogenic earthquakes such as Tohoku 2011, Illapel 2015, Mentawai 2010, Haida Gwaii 2012 (Romano et al. 2014; Lorito et al. 2015; Romano et al. 2016) have all shown substantial surface rupture. This is a particularly important feature in the case of tsunami hazard where shallow slip amplification can lead to increased tsunami wave height (Murphy et al. 2016; Scala et al. 2019b).

In summary, while the question as to whether rupture is pulseor crack-like remains open, large slip in the near surface does appear to occur and is important, particularly for tsunami generation. Therefore, we need to introduce a way to balance the systematic behaviour of a composite source model approach in the case of surface rupture.

In this paper, we propose two new techniques to tackle this issue. They are based on the principle that the slip can be reflected back onto the fault plane. Thus it negates the need to extend the fault above the surface. The first technique is based on the method of images and requires only a modest alteration of the original composite source model equations. It is only applicable to planar faults.
The second involves a multistage grid based technique commonly used in calculating traveltimes in seismology (Vidale 1990; Podvin \& Lecomte 1991; Noble et al. 2014). It can be applied to non-planar complex fault geometries.

\section{SURFACE RUPTURE: PLANAR FAULTS}

The composite source model involves the placement of a large number of circular slip functions, that is subevents, on the fault plane (Frankel 1991; Zeng et al. 1994). The size- frequency relationship of these subevents are power law in nature and were originally described as uniform slip distributions where the slip was proportional to the radius of the subevent $(\delta \propto r)$. Ruiz et al. $(2007,2011)$ built on this model by changing the slip function for the subevents from one that is uniform to an Eshelby crack (Eshelby 1957):

$u(r)= \begin{cases}\frac{24}{7 \pi} \frac{\Delta \sigma}{\mu} \sqrt{1-\frac{r^{2}}{R^{2}}} & r \leq R \\ 0 & r>R .\end{cases}$

In the eq. (1), $R$ is the radius of the subevent [at position $\left(x_{o}, y_{o}\right)$ ] and $r$ is radial distance from the centre of the subevent to any position on the fault plane. When one of these circular subevents crosses the boundary of the fault [i.e. $\sqrt{\left(x_{b}-x_{o}\right)^{2}+\left(y_{b}-y_{o}\right)^{2}}<R$ where $\left(x_{b}, y_{b}\right)$ is the nearest point on the fault boundary to the centre of the 

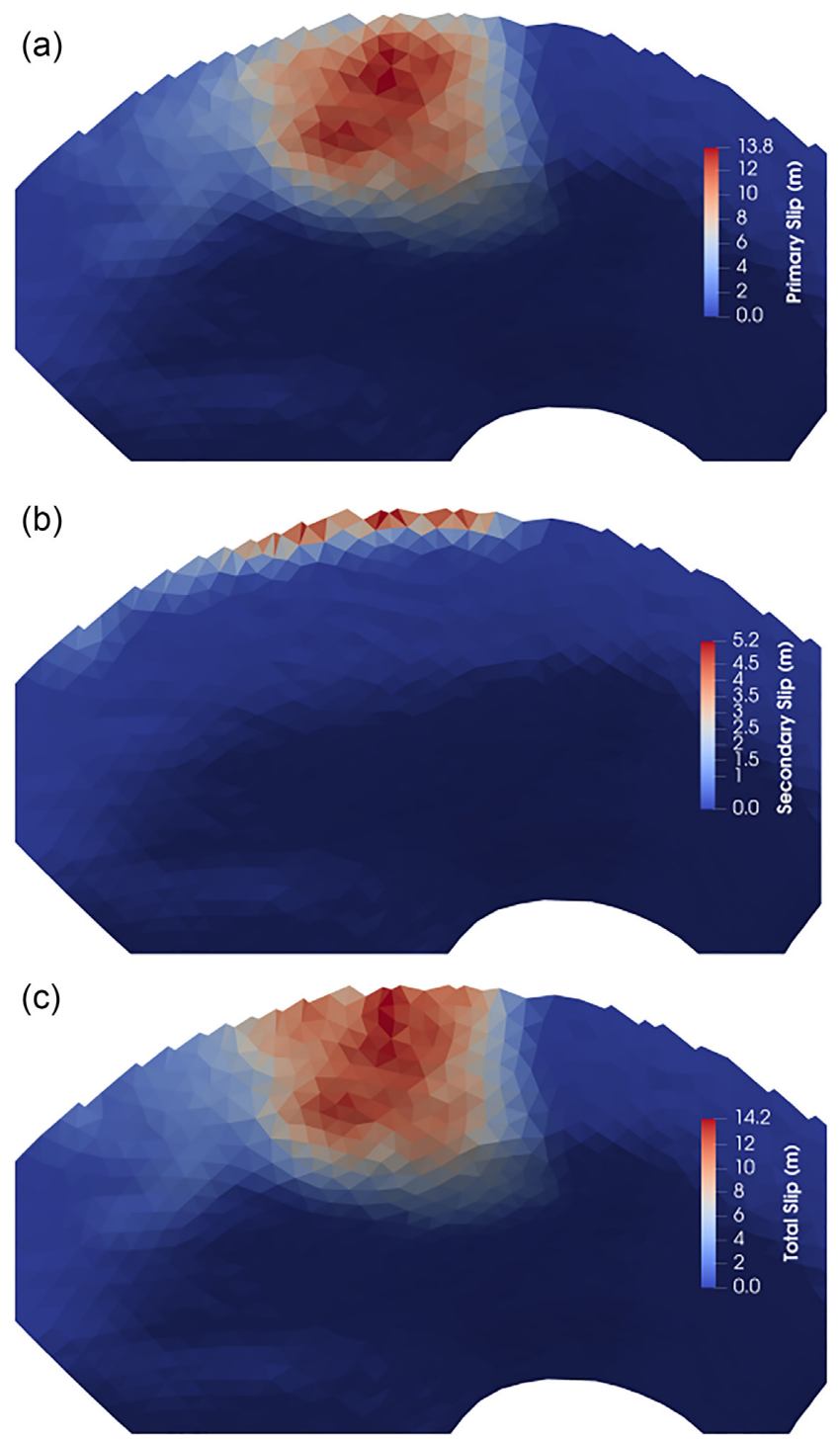

Figure 5. Example of surface rupture for $M 8.5$ earthquake on the Scotia subduction slab. Panel (a) is the slip distribution obtained when using only the initial subevents and not accounting for any reflections from the free surface. Panel (b) is the slip contribution from all the secondary sources $\left[u_{s}\left(r^{\prime \prime}\right)\right.$ in eq. (4)] in the cases where the initial subevent had a boundary that extended above the surface. Panel (c) is the final slip on the fault containing both the initial subevents and the secondary reflected ones.

subevent] a new subevent centre is randomly chosen. This procedure allows the slip to naturally taper to zero at the fault boundary.

In order to generate slip up to the surface this condition is relaxed so that subevents with radii extending across the surface are allowed. As mentioned in the introduction, this provides a mean of generating surface rupture. However the slip distributions tend to systematically decrease near the surface (Murphy et al. 2016). This is due to the spatial asymmetric distributions of subevents whereby fault cells located in the vicinity of the surface only receive contributions of slip from subevents located at or below the surface and not above it. Moving down-dip the effect of the surface decreases as a buried point on the fault is receiving slip from progressively more subevents of varying sizes across a wider azimuthal range.

A remedy is to reflect the segments of the subevents that extend over the free surface back onto the fault plane. This technique does not require an extension of the fault plane but rather the addition of a secondary source that represents this reflected slip.

In the case of a planar fault where the free surface occurs along the $x$-axis (i.e. $y=0$ ) this can be achieved by using the method of images (Fig. 1). By placing an identical mirrored subevent centred at $\left(x_{o},-y_{o}\right)$ (assuming depth is positive) above the surface, that is an 'imaginary source'. This requires only a slight modification to original eq. (1) where the radius is altered to:

$r^{\prime}=\sqrt{\left(x-x_{o}\right)^{2}+\left(y+y_{o}\right)^{2}}$.

The slip at/beneath the surface from this imaginary source provides the reflected contribution to the total slip. The total slip, $u(x, y)$, for one subevent that contains reflected slip is given as:

$u(x, y)=u_{p}(r)+u_{i}\left(r^{\prime}\right)$,

where $u_{p}(r)$ is the slip contribution from the original subevent while $u_{i}\left(r^{\prime}\right)$ is the reflected contribution. A schematic of the location of the two subevents is provide in Fig. 1. The combination of the primary and imaginary sources now doubles the slip along the surface. An example of the different components in eq. (3) is given in Fig. 2.

\section{SURFACE RUPTURE: NON-PLANAR FAULTS}

The method of images described in the previous section provides an easy solution in the case of a planar fault where the shortest distance to the surface is perpendicular to it. In the case of a non-planar fault where the surface is no longer straight, use of the method of images becomes non-trivial. However computing distance or travel time from a technical point of view is the same. Therefore calculating the reflected distance from a boundary is similar to the issue of calculating the travel time from a particular reflector in a complex media (e.g. Podvin \& Lecomte 1991; Rawlinson \& Sambridge 2005). We introduce a multistage method that uses the trilateration technique (Herrero \& Murphy 2018), which computes the geodesic distance on a complex surface, to estimate the distance from a source after a reflection. To obtain this result we need to apply the technique to all the points defining the reflector twice. Consequently this method is called the 'multistage trilateration technique' (MSTT). Methods to solve this problem involve either setting the velocity below the reflector to zero (e.g. Podvin \& Lecomte 1991) or by resetting and recalculating the travel time in the layer above the reflector (e.g. Rawlinson \& Sambridge 2005). The MSTT method we apply for calculating the reflected difference off the free surface is based on the latter scheme with the reflector of interest situated along the edge of the mesh.

First, the nodes in the mesh making up the free surface were noted, henceforth referred to as the 'surface nodes'. Then, the distances were calculated from a point source, that is the centre of the subevent, to all other points on the mesh/grid - this is depicted in Fig. 3(a). The distance along the surface nodes are then saved (see Fig. 3b). Next, the distance is then recomputed across the mesh a second time using all the surface nodes as a line source. To ensure a correct computation, the distance is reset on all the nodes to their initial condition, with the exception of surface nodes which retain their distance value from the first iteration (Fig. 3c).

The strength of this technique is the ease of application with the repeated use of the same technique for calculating distance across the mesh. A disadvantage of the method is that the computation cost is doubled.

To compute the slip this reflected distance is substituted into eq. (1) in place of $r$. The slip function generated using the reflected 
distances, $r^{\prime \prime}$, is similar to the contribution $u_{i}\left(r^{\prime}\right)$ in eq. (3) and is added to the primary subevent in similar way:

$u(x, y)=u_{p}(r)+u_{s}\left(r^{\prime \prime}\right)$,

where $u_{s}\left(r^{\prime \prime}\right)$ is the contribution of the secondary source (i.e. the reflected source).

With a planar fault the two techniques can be tested against each other. In Fig. 4(a) the reflected distance for a point source at a distance of $25 \mathrm{~km}$ along strike and $6.5 \mathrm{~km}$ depth has been computed using the two techniques. In Fig. 4(b) a subevent with a radius of $25 \mathrm{~km}$ has been placed in the same location as in subplot 4(a) and the $u_{i}$ and $u_{s}$ in eqs (3) and (4) have been calculated and compared against each other. In both figures the error is normalized, see caption for more details. For this reason error in reflected slip in Fig. 4(b) is highest at the edge of the subevent (i.e. due to the slip approaching zero). In both tests the error is near to the numerical noise.

To demonstrate the accumulative contribution that the secondary source can potentially provide, a M8.5 earthquake with surface rupture was generated on the Scotia slab (Hayes et al. 2012). In Fig. 5 the contributions from all the primary $\left(u_{p}\right)$ and secondary $\left(u_{s}\right.$, i.e. reflected) slip have been summed up separately in subplots (a) and (b), respectively. Comparing the three subplots the secondary source provides a large slip component right at the surface which would not be possible to achieve if only the primary source was used (in some cells up to 40 per cent of the surface rupture is supplied by $u_{s}$ ).

\section{DISCUSSION}

With both techniques (i.e. MSTT and method of images), the surface has been treated as a mirror reflecting the slip at shallow depth. This is similar to Knopoff (1958) assumption where the surface is also treated as a mirror. However the assumption that rupture is crack-like is not being made in our case. Only the individual local subevents near the surface are being reflected but not the final slip across the fault. Dynamic simulations suggest that the amount of surface rupture is a function of fault dip and faulting mechanism (Oglesby et al. 1998; Nielsen 1998; Scala et al. 2019a). Such phenomena could be incorporated into this model by adding a multiplicative factor to the reflected slip contribution, thereby acting as a 'tuning factor'. It would require a more complete description of the effect that different geometries and faulting mechanism play on the slip.

A further step for the surface rupture model is the inclusion of variable rise time functions based on local subevent size, that is $\tau \propto R$ (Ruiz et al. 2007). Bilek \& Lay $(1998,1999)$ observed a systematic variation in the source duration with depth in subduction zones, suggesting earthquakes in such zones call for a careful depth dependent treatment. Again a depth dependent 'tuning factor' in this case for the rise-time could be added.

Fig. 5 highlights the contribution of the reflected slip on a single earthquake. To better quantify the trend due to the inclusion of the reflected slip, we have produced 500 stochastic slip distributions. As in Figs 5(a) and (c) two slip distributions from each simulation are generated: one containing only the initial subevents and the other containing both the initial and secondary slip sources. The initial slip sources represents the standard composite source model.

The mean slip per cell for the two groups of slip distributions, primary slip and the sum of primary and secondary slip, is displayed in Fig. 6. The depth profile shown in the top panel (Fig. 6c) highlights (a)

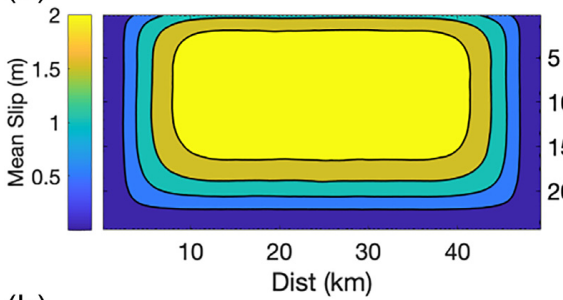

(b)

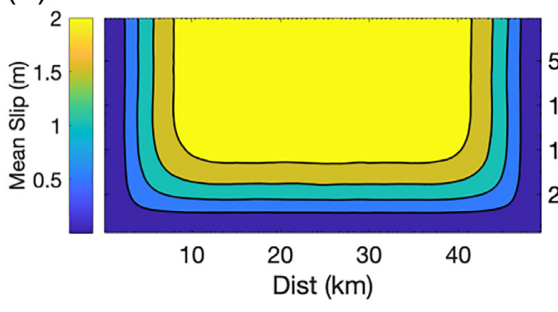

(d)

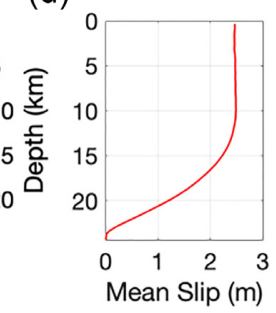

Figure 6. Spatial distribution of mean slip for 500 simulations excluding/including near surface slip reflection. Panels (a) and (b) depict the spatial distribution of average slip across the fault in the case where near surface reflected slip is excluded/included respectively. Panels (c) and (d) are crosssections of the average slip taken from subplots (a) and (b) respectively at $25 \mathrm{~km}$ along strike.

the issue that this paper aims to address: a systematic and relative decrease of the slip close to the surface, when we use a composite source model. The lower panel demonstrates the validity of the correction we have applied. The depth profile (Fig. 6d) exhibits a constant average slip value from the centre of the fault up to the surface.

Such a feature is particularly important for the probabilistic hazard assessment where large numbers of earthquake sources are used. The family of slip distributions used in such models should, if possible, converge to a spatially uniform distribution across the fault. Consequently if a slip needs to vary spatially (e.g. accounting for recent earthquakes, coupling, depth dependency, etc.) this can be added as a subsequent step on the corrected composite source model.

The choice of the type of probability density function (PDF) used in the placement of the subevents on the fault can alter the distribution of the slip. Up until now (i.e. Figs 5 and 6) the subevents have been placed on the fault plane using an uniform PDF. An alternative option is a Gaussian function (e.g. Cultrera et al. 2010; Akinci et al. 2017) as shown in Fig. 7(a) which can provide a first order constraint on the location of the largest slip (for e.g. compare the location of the peak of the Gaussian function in Fig. 7(a) with the location of maximum slip in Fig. 7(b). Achieving a spatially uniform average slip for a family of slip distributions using such a PDF is challenging. 1000 stochastic slip distributions were generated using a Gaussian PDF to place the subevents. The location of the maximum of the Gaussian was in turn randomly placed on the fault using a uniform PDF. All the other conditions are the same as those used in producing Fig. 6(b). However Fig. 7(d) exhibits again a systematic decrease in slip near the surface even if the surface rupture effect is corrected for. Therefore this feature is only due to the choice of the PDF. Calculating the average PDF used in the placement of subevents generates a function which is concentrated in the centre of the fault, see Fig. 7(c). This is similar to the coverage of the final slip distribution (compare to Fig. 7d). It demonstrates that the average a priori for our stochastic model, that is the PDF used to place the subevents, is representative, to a first order, of the characteristic output of the model. 

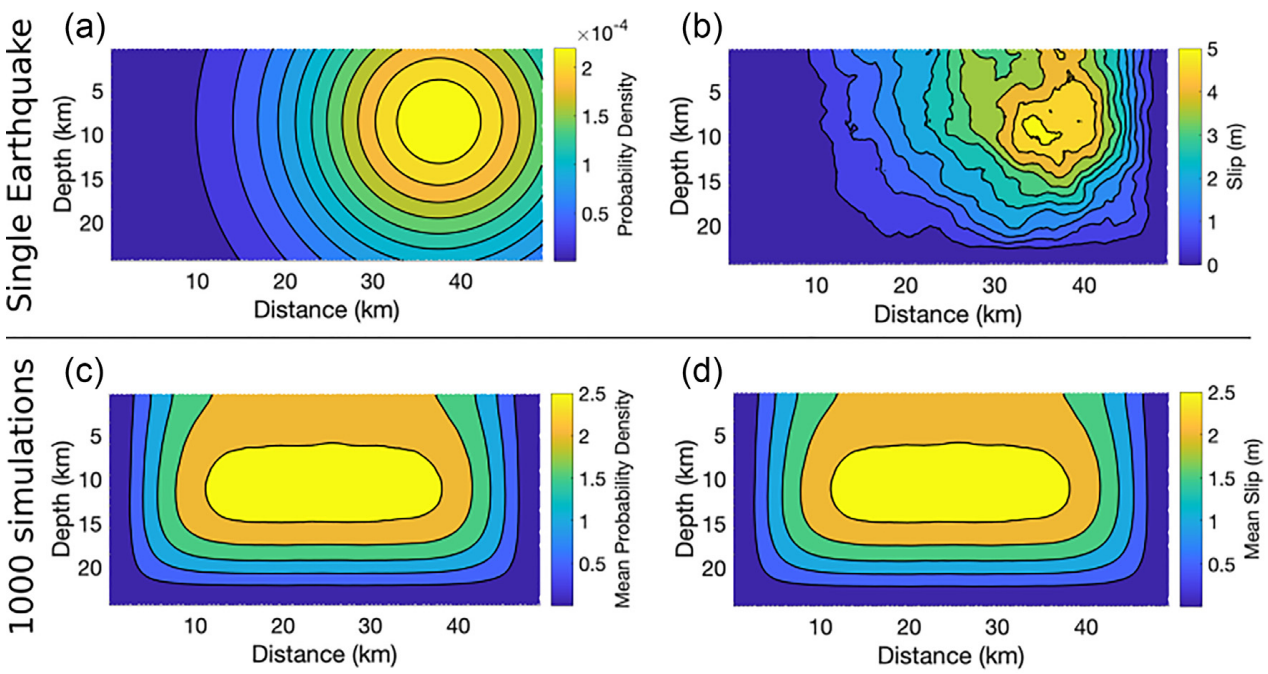

Figure 7. Different PDFs for defining subevent locations have different effects on the slip distributions both for a singular event and a family of simulations. Panel (a) is an example of a Gaussian function that has been used as a PDF for the location of subevents in the production of the slip distribution in (b). Panel (c) is the mean PDF for 1000 simulations where a Gaussian function similar to that shown in subplot (a) was randomly placed on the fault. Panel (d) is the spatial distribution of mean slip for 1000 stochastic slip distributions produced using the randomly placed Gaussian functions.

An additional feature of using a Gaussian function is the need for more simulations to converge to a stable average slip distribution compared to a uniform function [500 earthquakes were required to produce Fig. 6(b) compared to 1000 in Fig. 7c].

In the case of probabilistic seismic hazard assessment or probabilistic tsunami hazard assessment a spatially uniform distribution of slip over a large family of earthquakes may be more desirable. Conversely, where the operator would like to constrain the location of maximum slip to a first order, a probability density function based on a Gaussian function may be more appropriate. However a nonuniform distribution will generate systematic spatial biases when a number of the slip distributions are summed together. Therefore choosing the probability density function used in the placement of subevents on the fault plane has consequences and requires careful analysis.

The inclusion of surface rupture translates into larger surface deformation which is particularly important in the case of tsunami generation. To demonstrate this, two slip distributions were produced, one with only the primary slip contribution (i.e. no surface rupture) and the second containing both primary and secondary slip contributions (which will be referred to as the 'surface rupture' case). A regular, planar, grid with a length of $60 \mathrm{~km}$, width of 30 $\mathrm{km}$ and a dip of $30^{\circ}$ hosting a magnitude 7 earthquake was used so that the triangles could be combined to create squares which were in turn used to calculate the surface deformation vector for a range of points using an analytical solution for a simplified half-space media (Okada 1992). In Fig. 8(c) the difference between the surface deformation between the two cases is given as a percentage based on the magnitude of the difference between the two deformation vectors divided by the magnitude of the deformation in the case of surface rupture. The formula is given as:

$$
100 * \frac{\sqrt{\left(d_{x}^{s}-d_{x}^{b}\right)^{2}+\left(d_{y}^{s}-d_{y}^{b}\right)^{2}+\left(d_{z}^{s}-d_{z}^{b}\right)^{2}}}{\sqrt{d_{x}^{s}+d_{y}^{s}+d_{z}^{s}}},
$$

where $d^{s}$ is the deformation in the case of surface rupture and $d^{b}$ is for the case containing only the primary slip contribution, subscripts denote the component. Fig. 8(c) demonstrates that the deformation shows substantial increase (i.e. above 20 per cent and up to 60 per cent) at distances of roughly $5 \mathrm{~km}$ from the fault trace. The orientation of the surface deformation vector also varies between the two earthquakes as well. For every deformation vector we apply the equation:

$$
\phi=\arccos \left(\frac{d_{y z}^{s} \cdot d_{y z}^{b}}{\left|d_{y z}^{s}\right|\left|d_{y z}^{b}\right|}\right)
$$

where the superscript corresponds to the deformation due to the case where only the primary slip source was used (i.e. 'b') or surface rupture was properly accounted for, 's'. $d_{y z}$ relates to the 2-D vector made up of the deformation in the $y z$ plane (i.e. the vertical - perpendicular to strike plane). Fig. 8(d) shows that there is $+5^{\circ}$ change of angle up over $10 \mathrm{~km}$ zone on the hanging wall side of the fault with the largest change occurring at the edge of the fault trace. While this is a single example, it demonstrates that accounting for surface rupture affects a sizeable zone around the fault trace, where both the magnitude and direction of the surface deformation changes. This is particularly important in tsunami generation where both the vertical and horizontal deformation (depending on the bathymetry) influences the tsunami source (Tanioka \& Satake 1996).

Ground motion may also be influenced by the presence of surface rupture. In the case of our stochastic slip distribution a $k^{-2}$ spectra is maintained when surface rupture is accounted for. This is described in more detail in Appendix A. Observations point to a relative decrease in amplitude around $1 \mathrm{~Hz}$ for ground motion recorded for surface rupturing earthquakes compared with buried cases with the hypothesis that it is due to different rupture processes (Kagawa et al. 2004). Therefore a combination of surface rupture and particular choices in the source time function with depth may be required in order to match such observations. A future aim is to investigate the effect of surface rupture on ground motion which would require the construction of stochastic source time functions which is beyond the scope of this paper. 

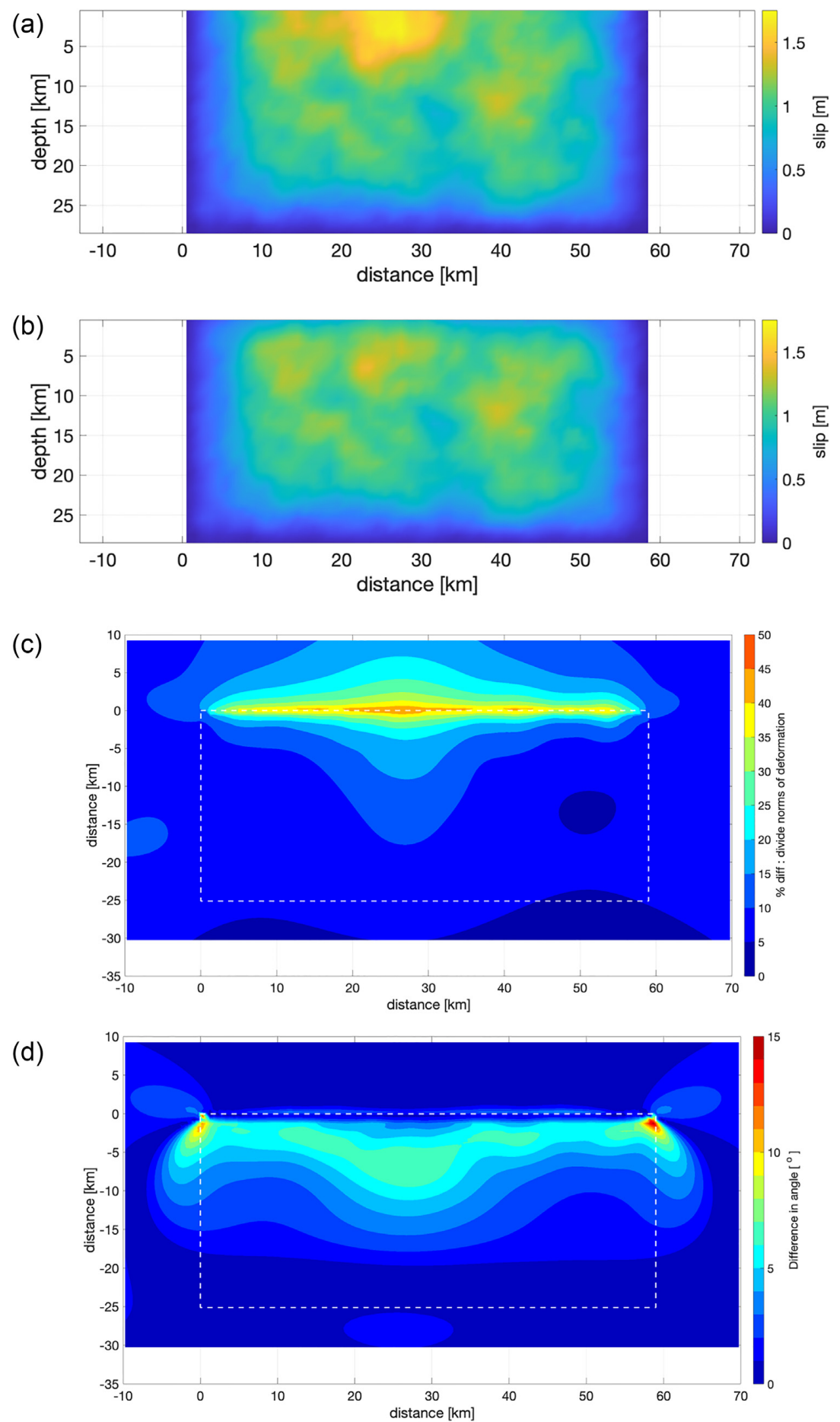

Figure 8. Comparison of surface deformation for two slip distributions with and without surface rupture for a $M 7$ reverse earthquake with a dip of $30^{\circ}$. Panels (a) and (b) are the two slip distributions, the first is the surface rupture case while the second contains only the primary slip. Panel (c) is the difference in the magnitude of the surface deformation between the two slip distributions. The percentage is calculated by subtracting the magnitude of the two surface deformations away from each other and dividing by the magnitude of the surface deformation in the case of the surface rupture. (d) The difference in angle between the two sets of deformation vectors. The angle is calculated from the vertical and perpendicular to the fault trace (i.e. the $y$-direction in the figure) components of the surface deformation. The yellow dashed line in subplots (c) and (d) represent the projection of the fault plane onto the surface.

\section{CONCLUSION}

The composite source model provides a technique for placing fractal slip on non-planar faults. A limitation of the methodology to date is a depth dependent trend that systematically underestimates nearsurface slip in the case of surface rupture. This behaviour is not always in agreement with some theories and observations. We have proposed two new techniques to correct this trend. In both cases the slip from subevents that extend over the surface have been reflected back on to the fault plane. In the first case, the method of images was used. This requires only a minor change to composite source model 
and works for planar faults. For non-planar faults, a multistage trilateration technique was applied. This technique requires that the fault is described by an unstructured mesh. The reflected slip calculated using the two different techniques is near identical on a planar fault.

Incorporation of the reflected slip generates much higher slip at the fault surface consistently. The choice of the probability density function for deciding the location of the subevents in the individual slip distributions should depend on the overarching objective of the stochastic modelling. If the rough location of the maximum slip is an a priori feature, a Gaussian function is more appropriate. If, on the other hand, the spatial distribution of slip is required to converge to an uniform level across the fault, an uniform probability density function should be used.

\section{RESOURCES}

The codes used to calculate the stochastic slip distribution are written in Fortran90. The code to compute the slip with surface rupture is available on Github at this address https://github.com/s-murfy/k $223 \mathrm{~d}$, while the code used to calculate distance across a non-planar surface which is used in the stochastic slip code can be found at this address https://github.com/andherit/trilateration. The codes used to produce the data used in all the figures have been put in the public domain. The geometry of the slab for Fig. 5 comes from the Slab1.0 database (Hayes et al. 2012) and may be found at this address https://earthquake.usgs.gov/data/slab/.

\section{ACKNOWLEDGEMENTS}

Figures has been created using Inkscape, Paraview (Ayachit 2015) and Matlab. We would like to thank the two anonymous reviewers for their comments which improved this manuscript. This research has been funded by the TSUMAPS-NEAM Project, cofinanced by the European Union Civil Protection Mechanism, Agreement Number: ECHO/ SUB/2015/718568/PREV26.

\section{REFER ENCES}

Akinci, A., Aochi, H., Herrero, A., Pischiutta, M. \& Karanikas, D., 2017. Physics - based broadband ground-motion simulations for probable $\mathrm{Mw} \geq 7.0$ earthquakes in the Marmara Sea Region (Turkey), Bull. seism. Soc. Am., 107(3), 1307-1323.

Andrews, D., 1980. A stochastic fault model 1. Static Case, J. geophys. Res., 85(B7), 3867-3877.

Ayachit, U., 2015. The ParaView Guide: A Parallel Visualization Application, Kitware Inc.

Bilek, S.L. \& Lay, T., 1998. Variation of interplate fault zone properties with depth in the Japan subduction zone, Science, 281(5380), 1175-1178.

Bilek, S.L. \& Lay, T., 1999. Rigidity variations with depth along interplate megathrust faults in subduction zones, Nature, 400(6743), 443-446.

Cultrera, G., Cirella, A., Spagnuolo, E., Herrero, A., Tinti, E. \& Pacor, F., 2010. Variability of kinematic source parameters and its implication on the choice of the design scenario, Bull. seism. Soc. Am., 100(3), 941-953.

Davies, G., Horspool, N. \& Miller, V., 2015. Tsunami inundation from heterogeneous earthquake slip distributions: evaluation of synthetic source models, J. geophys. Res., 120(9), 6431-6451.

Eshelby, J. D., 1957. The determination of the elastic field of an ellipsoidal inclusion, and related problems, Proc. R. Soc. A, 241, 376-396.

Frankel, A., 1991. High-frequency spectral falloff of earthquakes, fractal dimension of complex rupture, $\mathrm{b}$ value, and the scaling of strength on faults, J. geophys. Res., 96(B4), 6291-6302.
Goda, K., Yasuda, T., Mori, N. \& Mai, P.M., 2015. Variability of tsunami inundation footprints considering stochastic scenarios based on a single rupture model: application to the 2011 Tohoku earthquake, J. geophys. Res, 120 (6), 4552-4575.

Hanks, T.C., 1979. b values and $\omega^{-\gamma}$ seismic source models: implications for tectonic stress variations along active crustal fault zones and the estimation of high-frequency strong ground motion, J. geophys. Res., 84(B5), 2235-2242.

Hayes, G.P., Wald, D.J. \& Johnson, R.L., 2012. Slab1.0: a three-dimensional model of global subduction zone geometries, J. geophys. Res., 117(B1), B01302.

Heaton, T., 1990. Evidence for and implications of self-healing pulses of slip in earthquake rupture, Phys. Earth planet. Inter., 64, 1-20.

Herrero, A. \& Bernard, P., 1994. A kinematic self-similar rupture process for earthquakes, Bull. seism. Soc. Am., 84(4), 1216-1228.

Herrero, A. \& Murphy, S., 2018. Self-similar slip distributions on irregular shaped faults, Geophys. J. Int., 213(3), 2060-2070.

Kagawa, T., Irikura, K. \& Somerville, P.G., 2004. Differences in ground motion and fault rupture process between the surface and buried rupture earthquakes, Earth, Planets Space, 56(1), 3-14.

Knopoff, L., 1958. Energy release in earthquakes, Geophys. J. Int., 1(1), $44-52$.

Lavallée, D. \& Archuleta, R.J., 2003. Stochastic modeling of slip spatial complexities for the 1979 Imperial Valley, California, earthquake, Geophys. Res. Lett., 30(5), 1245.

Lorito, S., Romano, F. \& Lay, T., 2015. Tsunamigenic major and great earthquakes (2004-2013): source processes inverted from seismic, geodetic, and sea-level data, in Encyclopedia of Complexity and Systems Science, pp. 1-52, Springer Berlin Heidelberg.

Mai, P. \& Beroza, G., 2002. A spatial random field model to characterize complexity in earthquake slip, J. geophys. Res., 107(B11), ESE 10-1-ESE $10-21$.

Murphy, S. et al., 2016. Shallow slip amplification and enhanced tsunami hazard unravelled by dynamic simulations of mega-thrust earthquakes, Sci. Rep., 6, 1-12,

Nielsen, S.B., 1998. Free surface effects on the propagation of dynamic rupture, Geophys. Res. Lett., 25(1), 125-128.

Noble, M., Gesret, A. \& Belayouni, N., 2014. Accurate 3-D finite difference computation of traveltimes in strongly heterogeneous media, Geophys. J. Int., 199(3), 1572-1585.

Oglesby, D., Archuleta, R. \& Nielsen, S., 1998. Earthquakes on dipping faults: the effects of broken symmetry, Science, 280, $1055-1059$.

Okada, Y., 1992. Internal deformation due to shear and tensile faults in a half-space, Bull. seism. Soc. Am., 82(2), 1018-1040.

Podvin, P. \& Lecomte, I., 1991. Finite difference computation of traveltimes in very contrasted velocity models: a massively parallel approach and its associated tools, Geophys. J. Int., 105, 271-284.

Rawlinson, N. \& Sambridge, M., 2005. The fast marching method: an effective tool for tomographic imaging and tracking multiple phases in complex layered media, Explor. Geophys., 36(4), 341-350.

Romano, F. et al., 2014. Structural control on the Tohoku earthquake rupture process investigated by 3D FEM, tsunami and geodetic data, Sci. Rep., 4(5631), doi:10.1038/srep05631.

Romano, F., Piatanesi, A., Lorito, S., Tolomei, C., Atzori, S. \& Murphy, S., 2016. Optimal time alignment of tide-gauge tsunami waveforms in nonlinear inversions: application to the 2015 Illapel (Chile) earthquake, Geophys. Res. Lett., 43(21), 11 226-11235.

Ruiz, J., Baumont, D., Bernard, P. \& Berge-Thierry, C., 2007. New approach in the kinematic $k^{-2}$ source model for generating physical slip velocity functions, Geophys. J. Int., 171(2), 739-754.

Ruiz, J.A., Baumont, D., Bernard, P. \& Berge-Thierry, C., 2011. Modelling directivity of strong ground motion with a fractal, $k^{-2}$, kinematic source model, Geophys. J. Int., 186(1), 226-244.

Scala, A., Festa, G., Vilotte, J.-P., Lorito, S. \& Romano, F., 2019a. Wave interaction of reverse-fault rupture with free surface: numerical analysis of the dynamic effects and fault opening induced by symmetry breaking, J. geophys. Res., 124(2), 1743-1758. 
Scala, A. et al., 2019b. Effect of shallow slip amplification uncertainty on probabilistic tsunami hazard analysis in subduction zones: use of longterm balanced stochastic slip models, Pure appl. Geophys., pp. 1-24, doi:10.1007/s00024-019-02260-x.

Shimazaki, K., 1986. Small and large earthquakes: the effects of the thickness of seismogenic layer and the free surface, in Earthquake Source Mechanics, pp. 209-216, eds Das, S., Boatwright, J. \& Scholz, C.H., American Geophysical Union.

Song, S.G. \& Somerville, P., 2010. Physics-based earthquake source characterization and modeling with geostatistics, Bull. seism. Soc. Am., 100(2), 482-496.

Tanioka, Y. \& Satake, K., 1996. Tsunami generation by horizontal displacement of ocean bottom, Geophys. Res. Lett., 23(8), 861-864.

Vidale, J.E., 1990. Finite-difference calculation of traveltimes in three dimensions, Geophysics, 55(5), 521-526.

Wells, D.L. \& Coppersmith, K.J., 1994. New empirical relationships among magnitude, rupture length, rupture width, rupture area, and surface displacement, Bull. seism. Soc. Am., 84(4), 974-1002.

Zeng, Y.H., Anderson, J.G. \& Yu, G., 1994. A composite source model for computing realistic synthetic strong ground motions, Geophys. Res. Lett., 21(8), 725-728.

\section{APPENDIX: POWER-LAW SCALING OF SLIP DISTRIBUTION}

The high frequency radiation of the source is expected to be generated by a distributed roughness of the rupture process or a stopping phase. In the case of the fault boundary along the surface we expect no stopping phase to be generated. However, it is important to check if the high frequency distribution of the source parameters are changed by the introduction of reflected slip.

To check the power-law scaling of the slip distribution with surface rupture we generated a planar fault described by a uniform mesh. As the spatial discontinuity of the slip at the surface would introduce noise at all wavelengths, the slip distribution is mirrored along the surface (i.e. the fault area has now been doubled, see Fig. A1a). Fig. A1(b) shows that the slip spectrum for this mirrored fault contains a $k^{-2}$ scaling.

With the assumption of fault mirroring the only variation of the slip spectrum from a buried fault is at the lowest wavenumber which is doubled in amplitude. In reality this is just an artefact. The main point is the stability of the behaviour of slip spectrum at high wavenumbers in a kinematic framework.

\section{(a)}

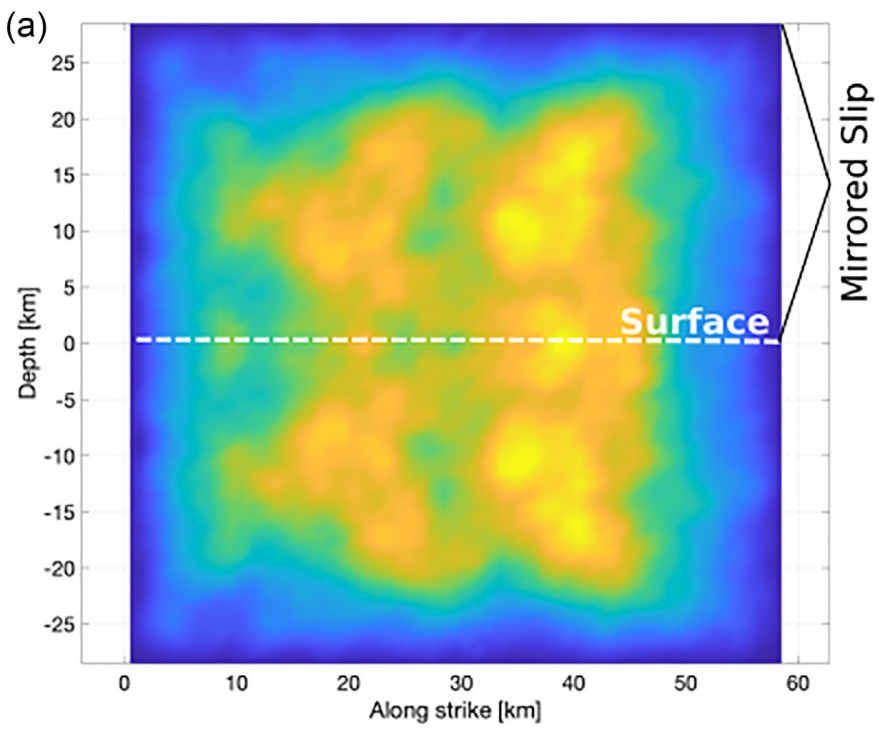

(b)

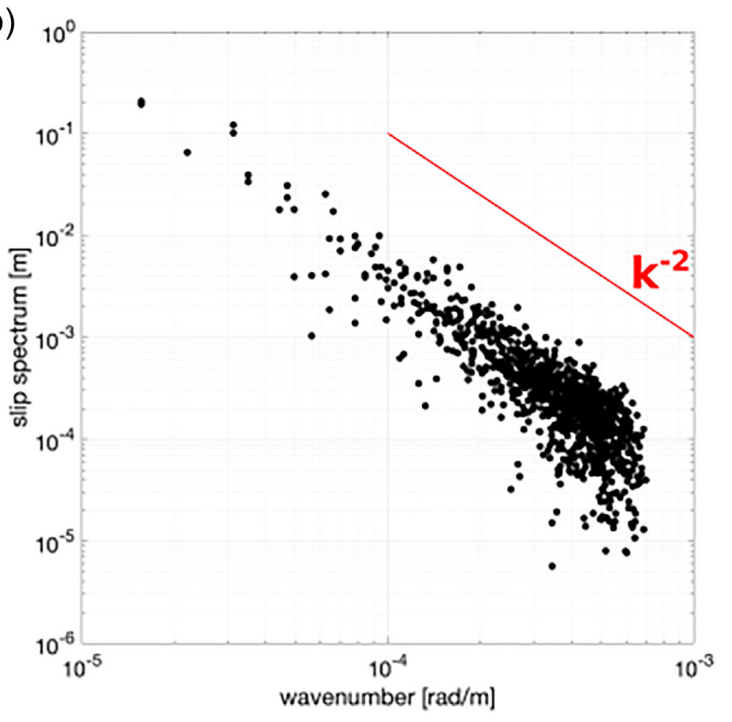

Figure A1. Checking the power-law scaling of a slip distribution with surface rupture. Panel (a) demonstrates how the slip distribution is mirrored around the surface. Panel (b) is the spectrum of the slip distribution shown in subplot (a). Each dot represents the amplitude spectra for each radial wavenumber. The red line represents a $k^{-2}$ slope for reference. The downgrading observed at high frequencies is a classical behaviour (e.g. Ruiz et al. 2007). 